\title{
Una mirada actualizada de los beneficios fisiológicos derivados del consumo de legumbres
}

\section{Revisiting the physiological benefits of legume consumption}

\section{RESUMEN}

Las legumbres han sido históricamente consideradas una apetecible fuente de proteínas y fibra. En estos tiempos caracterizados por una tendencia epidemiológica al sobrepeso y a la obesidad, corregir hábitos poco saludables es prioritario. La incorporación de legumbres al menú cotidiano como sustituto de carnes podría significar, a mediano y largo plazo, aminorar la prevalencia de enfermedades crónicas no-transmisibles. Este trabajo ofrece una mirada actualizada de algunos constituyentes claves presentes en las semillas de legumbres frecuentemente consumidas por la población chilena: porotos (Phaseolus vulgaris L.), lentejas (Lens culinaris L.); garbanzos (Cicer arietinum L.) y arvejas (Pisum sativum L.). Con una perspectiva realista, se expone el efecto del remojo y la cocción, ambos procesos simples utilizados frecuentemente en su preparación. Además, se ha considerado la digestión y fermentación como procesos claves en la liberación de compuestos bioactivos y su interacción con la microbiota residente en la porción distal del tracto gastrointestinal. Finalmente, estudios epidemiológicos en conjunto con datos experimentales permiten obtener una idea de los mecanismos que subyacen al impacto nutricional que tiene el consumo habitual de legumbres.

Palabras clave: Legumbres, Biodisponibilidad, Microbiota, Alimentos saludables.

\section{ABSTRACT}

Legumes have historically been considered a good source of proteins and fiber. Currently, there is an undeniable epidemiologic trend towards overweight and obesity; therefore, it is a priority to correct unhealthy habits. The incorporation of legumes to the daily menu, perhaps as a replacement for meat, could lead, in the short- or longterm, to a slowing in the prevalence of non-communicable chronic diseases. This work offers an updated perspective to some key constituents present in the legumes frequently consumed in the Chilean diet: beans (Phaseolus vulgaris L.), lentils (Lens culinaris L.), chickpeas (Cicer arietinum L.) and green peas (Pisum sativum L.). The effects of soaking and cooking, both simple processes widely used during its home preparation, along with digestion and fermentation,
Miltha Hidalgo, Vania Rodríguez y Omar Porras*.

Instituto de Nutrición y Tecnología de los Alimentos, Universidad de Chile.

Dirigir Correspondencia a: Omar Porras, PhD, Laboratorio de Biología Celular. Instituto de Nutrición y Tecnología de los Alimentos. Universidad de Chile, Av. El Líbano 5524, Macul, Santiago, Chile. E-mail:omar.porras@inta.uchile.cl

are crucial in the release of bioactive compounds at the distal portion of gastrointestinal tract, which determines its interaction with resident microbiota. In conclusion, epidemiological studies together with experimental data offer an integral view of subjacent mechanisms of nutritional impact by regular consumption of legumes.

Key words: Legumes, Bioavailability, Microbiota, Healthy foods.

\section{INTRODUCCIÓN}

La domesticación de algunos miembros de la familia de las Leguminosae se remonta a la edad de bronce y 
actualmente, forman parte esencial de la dieta moderna (i.e. dieta mediterránea) ${ }^{1}$. El desarrollo tecnológico y demográfico experimentado por cada región del globo ha moldeado dramáticamente sus patrones dietarios como lo demuestra una evaluación exhaustiva de los últimos 20 años².

En Chile, la producción de legumbres ha disminuido notablemente en estos últimos 30 años $^{3}$. Por otra parte, su consumo ha experimentado una disminución moderada, pero sostenida en el tiempo. En el caso de los porotos (Phaseolus vulgaris L.), su consumo ha disminuido un $12 \%$ en los últimos 5 años, un patrón que también se presenta en el consumo de lentejas y garbanzos ${ }^{4}$. Contra-intuitivamente, las importaciones combinadas de legumbres congeladas y secas han experimentado un incremento explosivo desde el año 2012 alcanzando un régimen estable por sobre las 900 toneladas/año en estos dos últimos años. A pesar de este panorama local, el consumo de legumbres por la población chilena es bastante satisfactorio; un estudio sistemático de los cambios experimentados en los patrones dietarios entre los años 1990 y 2010 realizado sobre 187 países posicionó a Chile dentro de los 10 países que consumen más legumbres, con un promedio que oscila entre 25-30 gramos diarios ${ }^{2}$.

Hoy en día, adoptar un patrón dietario más saludable implica favorecer la ingesta de alimentos mínimamente procesados. En este sentido, las legumbres poseen características en su composición que, al interactuar con el organismo durante la digestión y absorción, permiten destacar su consumo como un hábito alimentario saludable y, por consiguiente, recomendable para la población. El trabajo desarrollado a continuación expone evidencia científica actualizada de la base de datos de biomedicina del Instituto Nacional de la Salud de los Estados Unidos (NCBI, US), para entender cómo los fenómenos de digestión y fermentación conducen a la absorción de metabolitos con actividad biológica relevante que respalda algunos efectos beneficiosos descritos en estudios epidemiológicos.

\section{Composición y digestibilidad}

Las semillas de legumbres albergan un contenido importante de almidones y fibra, que en conjunto representan más del $50 \%$ de su composición. El contenido proteico le sigue con una representación de un 10 a 15\%, sin embargo, existen algunas especies de legumbres, como el poroto Lylongyihao jiadou, cuya abundancia en proteínas alcanza un $25 \%$. Las legumbres poseen un discreto contenido de lípidos, siendo los garbanzos los representantes de las leguminosas con mayor contenido lipídico, el cual no excede un 7\%, (Tabla 1).

El almidón contenido en semillas de porotos (Phaseolus vulgaris $L$.) está compuesto principalmente por polímeros de amilosa y amilopectina, que de acuerdo a su estructura ofrecen distinta resistencia a la degradación enzimática. Esta característica permite clasificarlos como rápidamente digeribles (amilopectina) hasta los resistentes a la digestión (amilosa). A pesar del alto contenido de almidones resistentes o de lenta digestión presentes en las legumbres crudas, el proceso de cocción es suficiente para transformar casi la totalidad de almidones en rápidamente digeribles ${ }^{6}$.

Durante la preparación convencional de legumbres, que incluye remojo y cocción, proteínas abundantes y solubles como la albúmina, y otras como las vicilinas, convicilinas y leguminas, consideradas globulinas, son liberadas de su matriz. A estas proteínas se les han adjudicado algunos efectos fisiológicos que incluyen la disminución de la colesterolemia, reducción de la glicemia postprandial, y efectos anti-hipertensivos y anti-cancerígenos ${ }^{7}$. Por ejemplo, algunos péptidos derivados de la fracción no digerible del poroto común (GLTSK, LSGNK, GEGSGA, MPACGSS y MTEEY) han sido evaluados por su capacidad anti proliferativa en una línea celular de carcinoma de colon ${ }^{8}$. Estos péptidos también son capaces de inhibir a la acetilcolinesterasa (ACE) con IC50 que van desde 65 a $200 \mu \mathrm{M}$, actividad que podría estar relacionada con la modulación de la presión arterial, y con la capacidad de neutralizar algunos radicales libres ${ }^{9}$.

El contenido de fibra presenta alta variabilidad entre las especies y variedades evaluadas, lo cual se refleja en un amplio rango de contenido de fibra total que oscila entre $1-38 \%$ para los garbanzos y $1-34 \%$ para las lentejas (Tabla 2). La fibra con mayor abundancia corresponde a la fibra insoluble ${ }^{5,10,11}$, la cual no es fermentable y por consiguiente, actúa como material de arrastre favoreciendo el tránsito intestinal al aumentar el volumen fecal ${ }^{12}$. La fibra soluble, por otra parte, se considera de relevancia fisiológica, debido a que participa directamente en la fermentación colónica ${ }^{13-15}$ y juega un papel protector del epitelio intestinal, un efecto observado en ratones con úlceras antrales ${ }^{16}$, cuyo efecto citoprotector estaría mediado por la pectina, que fomenta la secreción de mucina en el yeyuno de ratas al aumentar la expresión de Muc2 ${ }^{17}$.

\section{Micronutrientes y compuestos bioactivos}

El consumo de legumbres conlleva la ingesta de un número importante de micronutrientes que incluyen minerales y vitaminas, cuya abundancia está señalada en la tabla 3a y 3b. La presencia de minerales está acompañada del anión fosfato, que forma parte estructural de biomoléculas como el ácido fítico o mio-inositol hexafosfato, IP6, el cual podría formar complejos insolubles capaz de secuestrar cationes divalentes y, en consecuencia, disminuir la biodisponibilidad de $\mathrm{Fe}^{2+}$ y $\mathrm{Zn}^{2+}$, lo cual lo posiciona como un "antinutriente" ${ }^{\prime 18}$. Sin embargo, esta noción es discutible, ya que existen estudios que apoyan un rol negativo del ácido fítico, mientras que otros la descartan ${ }^{19,20}$. Afortunadamente, el remojo y la cocción a presión son suficientemente efectivos para reducir el contenido de IP6 y transformarlo en IP5, lo cual contribuye a una mayor captación de hierro por parte de células Caco-2, sugiriendo que un menor contenido de ácido fítico, ácido oxálico y polisacáridos complejos mejora la biodisponibilidad de micronutrientes ${ }^{21,22}$.

El contenido de polifenoles totales ha sido cuantificado por el método Folin-Ciocalteu en numerosas variedades de legumbres. En el caso de los porotos, estos análisis han 
Tabla 1

Macronutrientes en legumbres crudas y cocidas (g/100 gramos de semillas)

\begin{tabular}{|c|c|c|c|c|c|c|c|c|}
\hline \multirow{2}{*}{$\begin{array}{l}\text { Especie } \\
\text { (Nombre científico) }\end{array}$} & \multicolumn{2}{|c|}{ Proteínas } & \multicolumn{2}{|c|}{ Hidratos de carbono } & \multicolumn{2}{|c|}{ Lípidos } & \multicolumn{2}{|c|}{ Kcalorías } \\
\hline & Crudo & Cocido & Crudo & Cocido & Crudo & Cocido & Crudo & Cocido \\
\hline $\begin{array}{l}\text { Garbanzo } \\
\text { (Cicer arietinum) }\end{array}$ & $\begin{array}{l}21,2^{\mathrm{a}} \\
19,3^{\mathrm{b}} \\
18,2^{\mathrm{c}} \\
28,3^{\mathrm{d}} \\
20,5^{\mathrm{h}} \\
23,6^{\mathrm{i}}\end{array}$ & $\begin{array}{c}8,8^{\mathrm{b}} \\
6,6^{\mathrm{c}} \\
19,8^{\mathrm{d}} \\
8,9^{\mathrm{h}} \\
23,2^{\mathrm{i}} \\
9^{\mathrm{n}}\end{array}$ & $\begin{array}{l}45,5^{\mathrm{a}} \\
60,5^{\mathrm{b}} \\
57,7^{\mathrm{c}} \\
64,1^{\mathrm{d}} \\
60,7^{\prime}\end{array}$ & $\begin{array}{l}27,4^{b} \\
21,6^{\mathrm{c}} \\
70,4^{\mathrm{d}}\end{array}$ & $\begin{array}{l}5,4^{\mathrm{a}} \\
6,1^{\mathrm{b}} \\
6,2^{\mathrm{c}} \\
4,3^{\mathrm{d}} \\
5,0^{\mathrm{g}} \\
6,0^{\mathrm{h}} \\
6,5^{\mathrm{i}} \\
6,0^{\mathrm{l}} \\
5,7^{\mathrm{q}}\end{array}$ & $\begin{array}{l}2,6^{\mathrm{b}} \\
2,0^{\mathrm{c}} \\
7,2^{\mathrm{d}} \\
2,6^{\mathrm{h}} \\
6,2^{\mathrm{i}}\end{array}$ & $\begin{array}{l}340^{\mathrm{a}} \\
364^{\mathrm{b}} \\
359^{\mathrm{c}} \\
408^{\mathrm{d}} \\
378^{\mathrm{h}}\end{array}$ & $\begin{array}{l}163^{\mathrm{b}} \\
131^{\mathrm{c}} \\
426^{\mathrm{d}} \\
164^{\mathrm{h}}\end{array}$ \\
\hline $\begin{array}{l}\text { Lenteja } \\
\text { (Lens culinaris) }\end{array}$ & $\begin{array}{l}25,4^{\mathrm{a}} \\
28,1^{\mathrm{b}} \\
24,0^{\mathrm{c}} \\
22,0^{\mathrm{o}} \\
23,8^{\mathrm{q}}\end{array}$ & $\begin{array}{c}9,0^{\mathrm{b}} \\
7,4^{\mathrm{c}} \\
22,5^{\mathrm{o}}\end{array}$ & $\begin{array}{l}49,3^{a} \\
56,8^{b} \\
57,4^{c}\end{array}$ & $\begin{array}{l}20,2^{b} \\
24,6^{c}\end{array}$ & $\begin{array}{l}1,8^{\mathrm{a}} \\
1,0^{\mathrm{b}} \\
1,3^{\mathrm{c}} \\
0,7^{\mathrm{o}} \\
1,0^{\mathrm{q}}\end{array}$ & $\begin{array}{l}0,4^{\mathrm{b}} \\
0,5^{\mathrm{c}} \\
1,5^{\mathrm{o}}\end{array}$ & $\begin{array}{c}337^{\mathrm{a}, \mathrm{b}, \mathrm{c}} \\
444^{\circ} \\
304^{\mathrm{q}}\end{array}$ & $\begin{array}{l}116^{b} \\
133^{c} \\
447^{o}\end{array}$ \\
\hline
\end{tabular}

a) Legumbres. Organización de las Naciones Unidas para la Alimentación y la Agricultura. 2016. www.fao.org.

b) Jury G. Urteaga C. Porciones de Intercambio y Composición Química de los Alimentos de la Pirámide Alimentaría Chilena, INTA, Universidad de Chile. 1997.

c) Gattás V. Guía de la composición nutricional de alimentos naturales, de la industria y preparaciones chilenas habituales. INTA, Universidad de Chile. 2 da. Ed. 2011.

d) Sarmento A, Barros L, Fernandes Â, Carvalho AM, Ferreira IC. J Sci Food Agric 2015; 95: 179-185.

e) Kan L, Nie S, Hu J, Wang S, Cui SW, Li Y, Xu S, Wu Y, Wang J, Bai Z, Xie M. Food Chem Toxicol 2017; 467-477.

f) Multari S, Neacsu M, Scobbie L, Cantlay L, Duncan G, Vaughan N, Stewart D, Russell WR. J Agric Food Chem 2016 ; 64: $7800-7811$.

g) Ryan E, Galvin K, O’Connor TP, Maguire AR, O’Brien NM. Plant Foods Hum Nutr 2007; 62: 85-91.

h) Wallace TC, Murray R, Zelman KM. Nutrients 2016; 8.

i) Alajaji S, El-Adawy T. J Food Comp Anal 2006; 19: 806-812.

j) Campos-Vega R, Oomah D, Loarca-Piña G, Vergara-Castañeda H. Foods 2013; 2: 374-392.

k) Collar C, Jiménez T, Conte P, Fadda C. Carbohydrate Polym 2014; 113: 149-158.

I) Jukanti AK, Gaur PM, Gowda CLL, Chibbar RN. Brit J Nutr 2012; 108: S11-S26.

m) Martian-Cabrejas MA, Sanfiz B, Vidal A, Mollá E, Esteban R, López-Andréu FJ. J. Agric. Food Chem 2004; 52: $261-266$.

n) Messina V. Am J Clin Nutr 2014; 100: 437S-42S.

o) Poelaert C, Despret X, Sindic M et al. J. Agric Food Chem 2017; 65: 435-444.

p) Simons CW, Hall III C, Tulbek M, Mendis M, Heck T, Ogunyemi S. J Sci Food Agric 2015; 95: $2287-2291$.

q) Thorne MJ, Thompson L, Jenkins D. Am J Clin Nutr 1983; 38: 481-488. 
Tabla 2

Contenido de fibra en legumbres crudas y cocidas por cada 100 gramos

\section{Especie \\ (Nombre científico)}

Poroto

(Phaseolus vulgaris)

\section{Crudo \\ Fibra (g) \\ Cocido}

$15,4^{\mathrm{b}}$

$24,5^{g}$

$25,8^{\mathrm{i}}$

$31^{\circ}$

$37-41^{\circ}$

$25,4^{9}$

$40-42^{\circ}$
Fibra insoluble (g)

$4,9^{b}$

\section{Fibra soluble (g) \\ Crudo \\ Cocido}

$7,7^{8}$

\begin{tabular}{|c|c|c|}
\hline $17^{g}$ & & $7,7^{8}$ \\
\hline $25-36^{h, j}$ & $31-41^{\mathrm{h}, \mathrm{j}}$ & $0,3-1,6^{h, j}$ \\
\hline $13,9^{i}$ & & $7,7^{i}$ \\
\hline $8^{p}$ & & $13,5^{\circ}$ \\
\hline
\end{tabular}

$5,5-14^{\mathrm{h}, \mathrm{j}}$

$13,5^{\circ}$

\begin{tabular}{|c|c|c|c|c|c|c|}
\hline $\begin{array}{l}\text { Garbanzo } \\
\text { (Cicer arietinum) }\end{array}$ & $\begin{array}{c}12,4^{\mathrm{a}} \\
6,8^{\mathrm{b}} \\
6,2^{\mathrm{c}} \\
\\
21,8^{\mathrm{i}} \\
7,3^{\mathrm{j}} \\
12,2^{\prime} \\
17,4^{\mathrm{n}} \\
15^{\mathrm{q}}\end{array}$ & $\begin{array}{c}6,8^{\mathrm{b}} \\
1,3^{\mathrm{c}} \\
38,4^{\mathrm{e}} \\
17,4^{\mathrm{k}} \\
7,6^{\mathrm{l}}\end{array}$ & $15,8^{\mathrm{i}}$ & $\begin{array}{c}5,7^{\mathrm{d}} \\
34,5^{\mathrm{e}}\end{array}$ & $3,5^{\mathrm{i}}$ & $\begin{array}{l}3,8^{\mathrm{d}} \\
3,5^{\mathrm{e}}\end{array}$ \\
\hline $\begin{array}{l}\text { Lenteja } \\
\text { (Lens culinaris) }\end{array}$ & $\begin{array}{c}10,7^{\mathrm{a}} \\
12^{\mathrm{b}} \\
2,9^{\mathrm{c}} \\
\\
20,1^{\mathrm{i}} \\
7,9^{\mathrm{j}} \\
11,7^{\circ}\end{array}$ & $\begin{array}{c}5,05^{\mathrm{b}} \\
1^{\mathrm{c}} \\
34,2^{\mathrm{e}}\end{array}$ & $13,6^{\mathrm{i}}$ & $3,7^{e}$ & $3,2^{i}$ & $30,5^{\mathrm{e}}$ \\
\hline $\begin{array}{l}\text { Arveja seca } \\
\text { (Pisum sativum) }\end{array}$ & $\begin{array}{c}26^{\mathrm{a}} \\
4^{\mathrm{c}} \\
\\
24,6^{\mathrm{i}} \\
14,6^{\mathrm{m}}\end{array}$ & & $\begin{array}{c}8,7^{f} \\
16^{i} \\
8,5^{\mathrm{m}}\end{array}$ & & $\begin{array}{c}0,08^{f} \\
3,9^{i} \\
6,1^{m}\end{array}$ & \\
\hline $\begin{array}{l}\text { Haba seca } \\
\text { (Vicia faba) }\end{array}$ & $\begin{array}{c}26,3^{\mathrm{a}} \\
1,6^{\mathrm{b}} \\
9,1^{\mathrm{c}} \\
25^{\mathrm{o}}\end{array}$ & & $9,4^{f}$ & & $0,56^{f}$ & \\
\hline
\end{tabular}

a) Legumbres. Organización de las Naciones Unidas para la Alimentación y la Agricultura. 2016. www.fao.org.

b) Jury G. Urteaga C. Porciones de Intercambio y Composición Química de los Alimentos de la Pirámide Alimentaría Chilena, INTA, Universidad de Chile. 1997.

c) Gattás V. Guía de la composición nutricional de alimentos naturales, de la industria y preparaciones chilenas habituales. INTA-Universidad de Chile. 2 da. Ed. 2011.

d) Surampudi P, Enkhmaa B, Anuurad E, Berglund E. Curr Atheroscler Rep 2016; 18: 75.

e) Hernández-Salazar M, Osorio-Diaz P, Loarca-Piña G, Reynoso-Camacho R, Tovar J, Bello-Pérez LA. J Sci Food Agric 2010 ; 90 : $1417-1422$.

f) Multari S, Neacsu M, Scobbie L, Cantlay L, Duncan G, Vaughan N, Stewart D, Russell WR. J Agric Food Chem 2016; 64: 7800-7811.

g) Martian-Cabrejas MA, Sanfiz B, Vidal A, Mollá E, Esteban R, López-Andréu FJ. J Agric Food Chem 2004; 52: $261-266$.

h) Campos-Vega R, Oomah D, Loarca-Piña G, Vergara-Castañeda H. Foods 2013; 2: 374-392.

i) Chen Y, McGee R, Vandemark G, Brick M, Thompson H. Nutrients 2016; 8: 829

j) Messina V. Am J Clin Nutr 2014; 100: 437S-42S.

k) Pittaway JK, Ahuja KD, Cehun M, Chronopoulos A, Robertson IK, Nestel PJ, Ball MJ. Ann Nutr Metab 2006; 50: $512-518$.

I) Wallace TC, Murray R, Zelman KM. Nutrients 2016; 8(12).

m) Collar C, Jiménez T, Conte P, Fadda C. Carbohydrate Polym 2014; 113: 149-158.

n) Jukanti AK, Gaur PM, Gowda CLL, Chibbar RN. Br J Nutr 2012; 108: S11-S26.

o) Nilsson A, Johansson E, Ekstrom L, Bjorck I. PLoS One 2013; 8: e59985.

p) Simons CW, Hall III C, Tulbek M, Mendis M, Heck T, Ogunyemi S. J Sci Food Agric 2015; 95: $2287-2291$.

q) Thorne MJ, Thompson L, Jenkins D. Am J Clin Nutr 1983; 38: 481. 


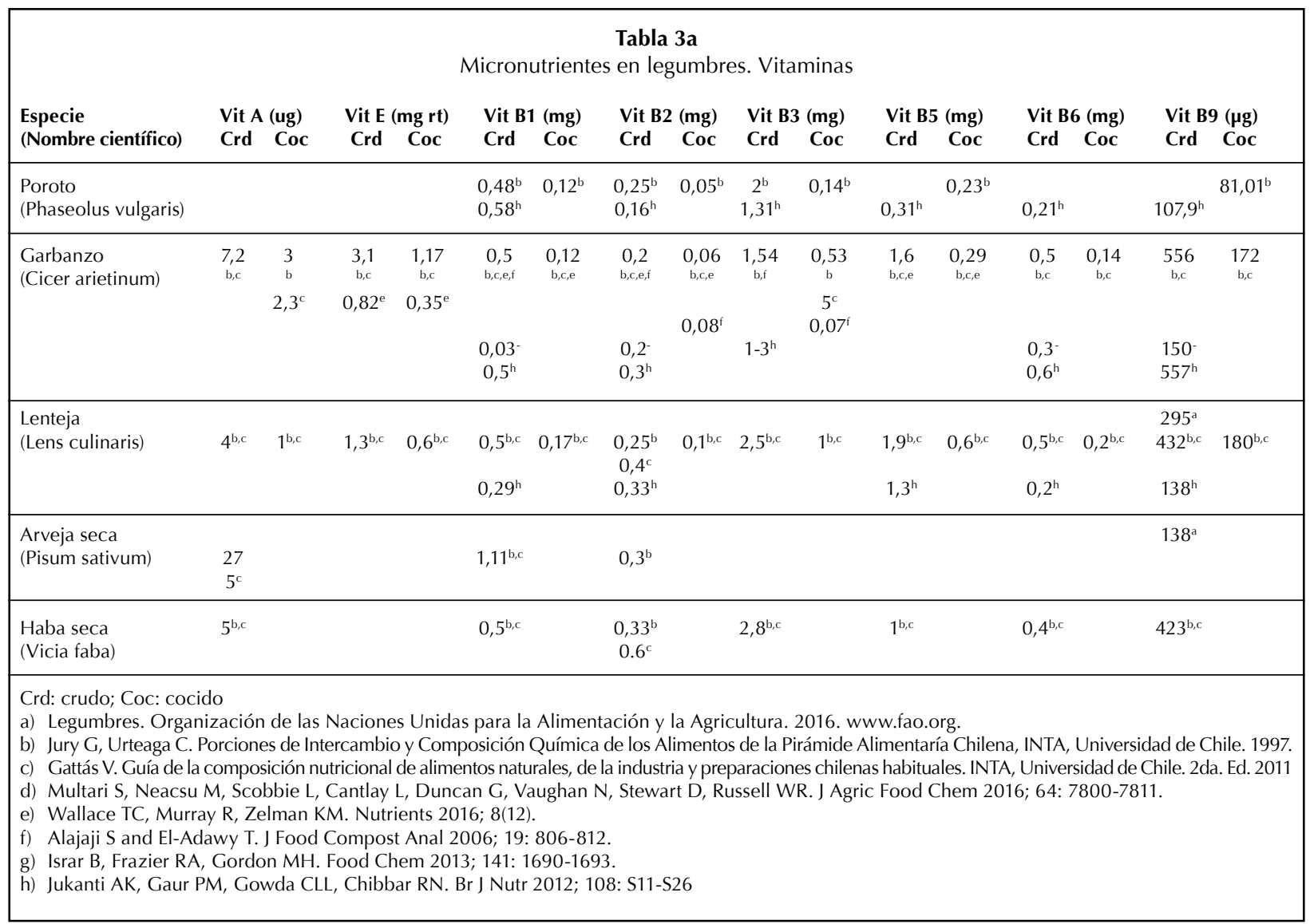

arrojado valores que van desde 0,1 a 3,8 mg (mg EAG/g, equivalentes de ácido gálico) que fluctúan de acuerdo al nivel de pigmentación que presenta la cubierta de la semilla, ${ }^{5,23,24}$. Estos valores son comparables a los reportados para el garbanzo (0,1 a 3,2 mg EAG), sin embargo, en lentejas estos compuestos alcanzan hasta $14 \mathrm{mg} \mathrm{EAG}^{23}$.

En el grupo de los polifenoles se pueden distinguir flavonoides y no flavonoides. Los primeros pueden ser subclasificados en antocianidinas, flavanoles, flavanonas, flavonoles, flavonas e isoflavonas; mientras que en el grupo de los no flavonoides se encuentran alcoholes monofenólicos, ácidos fenólicos y estilbenos. La cuantificación del contenido de flavonoides totales en porotos estima un contenido de 0,2 a 7,0 mg de flavonoides, expresado como mg de rutina por gramo de peso seco ${ }^{5}$. Otros análisis han identificado flavonoides específicos en garbanzos (quercetina y miricetina), habas (epicatequina, apigenina y luteolina), arvejas (apigenina y luteolina $)^{25}$. La presencia de ácidos fenólicos también ha sido determinada; en garbanzos predomina el ácido p-hidrobenzoico, como el principal ácido fenólico, mientras que, en arvejas secas, la fracción de ácidos fenólicos, compuesta por el ácido p-hidrobenzoico y el ácido protocatequínico, predomina por sobre los flavonoides; en el caso de las habas, sólo se detectó la presencia de ácido gálico ${ }^{25}$.
La abundancia de estos compuestos con potencial actividad antioxidante parece no ser afectada por la cocción ${ }^{24}$. El curso temporal de la presencia de flavonoides en la circulación sanguínea, determinada tras el consumo de porotos de soya, exhibe un patrón bifásico, con un incremento registrado una hora post-ingesta, seguido de una segunda elevación 3-4 veces mayor que la primera y observada 5-6 horas después de ingerir las legumbres. Se sugiere, con ello que, el mayor contenido de flavonoides se libera durante la fermentación colónica, desde la matriz vegetal que contiene a estos compuestos gracias a la acción de la microbiota ${ }^{26}$.

Una dieta rica en flavonoides se asocia con una reducción en el riesgo a padecer enfermedades crónicas no-transmisibles. Por ejemplo, la epicatequina administrada en ratas previno el daño renal inducido por una dieta alta en fructosa ${ }^{27}$, y en diversos modelos celulares de cáncer, la epicatequina y la protocatequina presentan algún grado de citotoxicidad en células neoplásicas ${ }^{28,29}$, un efecto que podría estar mediado por la inducción de apoptosis en líneas celulares de cáncer de colon y mama ${ }^{30,31}$. Es interesante destacar que el contenido total de polifenoles, asociado a la pigmentación de las semillas de porotos, también se traduce en la capacidad de inhibir la proliferación en líneas celulares con origen neoplásico, tales como Caco-2, A549 y MCF-724. 
Tabla 3b

Micronutrientes en legumbres. Minerales

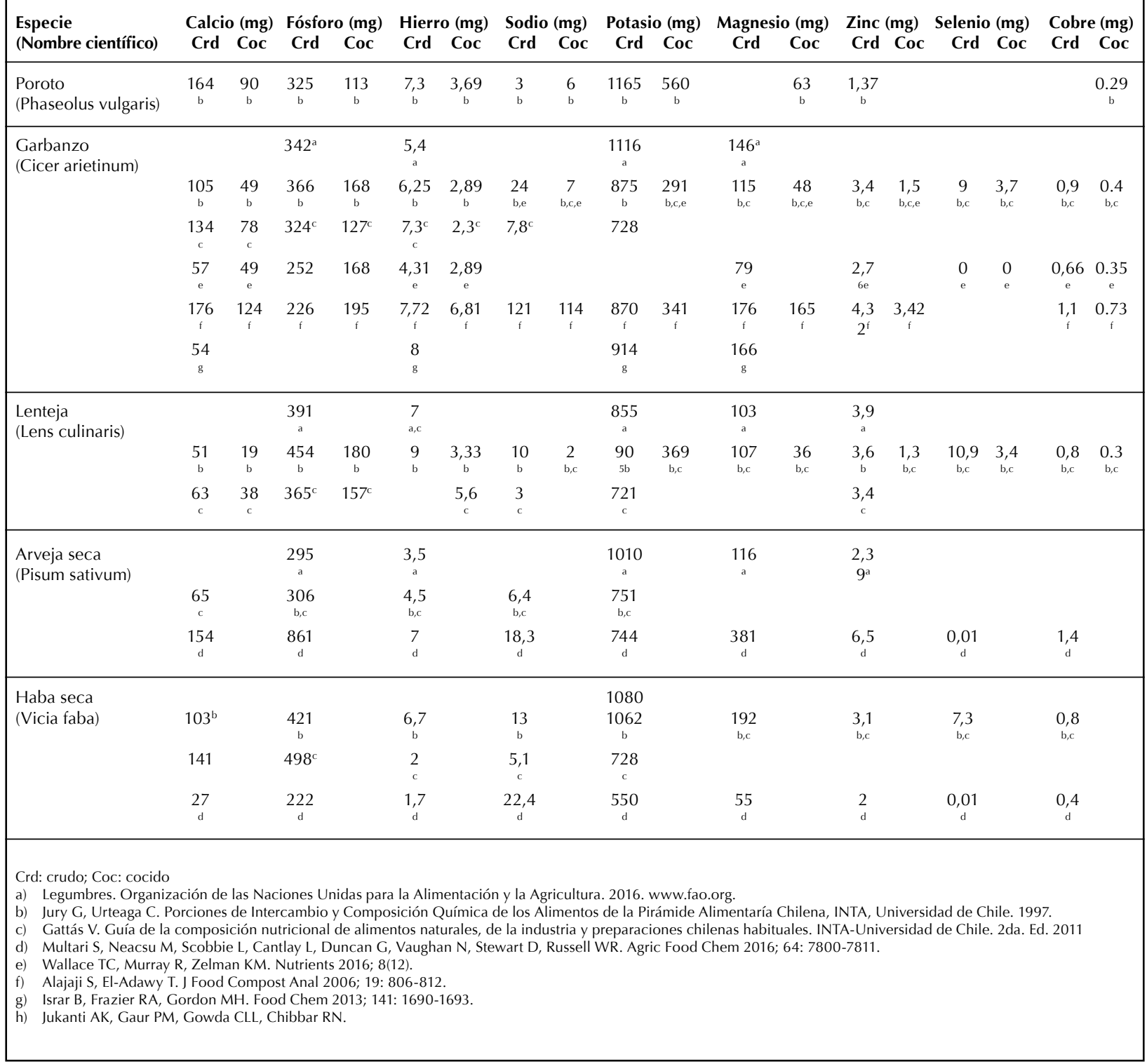

Los fitoesteroles corresponden a esteroles y estanoles, sus estructuras químicas comparten cierta relación con el colesterol, aunque presentan grupos adicionales como un grupo etilo (sitosterol) o un grupo metilo (campesterol) en su cadena lateral. El contenido total de fitoesteroles detectado en porotos fue de $134 \mathrm{mg}$, mientras que en arvejas fue de $242 \mathrm{mg}$ por cada 100 gramos de producto $\operatorname{seco}^{32}$ (Tabla 4). El sitosterol, el campesterol y el estigmasterol son los fitoesteroles comúnmente reportados en legumbres y el principal efecto benéfico para la salud consiste en reducir el riesgo cardiovascular mediado por una disminución en los niveles de lipoproteína de baja densidad (LDL) plasmáticos ${ }^{33}$. Por ejemplo, un estudio realizado con dos grupos diagnosticados con hipercolesterolemia, 30 sujetos sometidos a tratamiento con estatinas y otros $32 \mathrm{sin}$ tratamiento, fueron sometidos a la ingesta de grasa vegetal con fitoesterol (2,5 g/día) por 8 semanas, transcurrido este período se observó una disminución en los niveles de LDL plasmático de un $10-15 \%$ en ambos grupos ${ }^{34}$. Cabe señalar que, por cada $1 \%$ de reducción en los niveles de LDL plasmáticos se reduce la mortalidad cardiovascular en otro $1 \%{ }^{35}$. 


\begin{tabular}{|c|c|c|c|c|c|c|c|}
\hline $\begin{array}{l}\text { Especie } \\
\text { (Nombre científico) }\end{array}$ & $\begin{array}{c}\text { Ácido Fítico } \\
\text { (gr/kg) } \\
\text { Crudo }\end{array}$ & $\begin{array}{c}\text { Ácido Fítico) } \\
\text { (gr/kg } \\
\text { Cocido }\end{array}$ & $\begin{array}{l}\text { Polifenoles totales } \\
\text { (mg Eq de ácido } \\
\text { gálico/g peso seco) }\end{array}$ & $\begin{array}{l}\text { Ácidos fenólicos } \\
\text { (mg/kg peso seco) }\end{array}$ & B-sitosterol & $\begin{array}{c}\text { Fitoesteroles } \\
\text { (mg/100g) } \\
\text { Campesterol }\end{array}$ & Estigmasterol \\
\hline $\begin{array}{l}\text { Garbanzo } \\
\text { (Cicer arietinum) }\end{array}$ & $\begin{array}{c}12-22^{\mathrm{d}} \\
12,1 \pm 0,9^{\mathrm{e}} \\
16 \pm 0,4^{1}\end{array}$ & $\begin{array}{l}8,6 \pm 0,6^{\mathrm{e}} \\
15,4 \pm 0,4^{1}\end{array}$ & $\begin{array}{c}1,4 \pm 0,1^{\mathrm{a}} \\
0,13^{\mathrm{s}} \\
0,8 \pm 0,1^{\mathrm{t}}\end{array}$ & $27,5-107^{p}$ & $160 \pm 7^{q}$ & $21 \pm 1^{q}$ & $23 \pm 1^{9}$ \\
\hline $\begin{array}{l}\text { Haba seca } \\
\text { (Vicia faba) }\end{array}$ & & & & $23-138^{p}$ & & & \\
\hline $\begin{array}{l}\text { a) Poblaciones MJ } \\
\text { b) Davidsson L, D } \\
\text { c) Steiner T, Mose } \\
\text { d) Joshi-Saha A an } \\
\text { e) Alajaji S and El- } \\
\text { f) Humer E and Sc } \\
\text { g) Simons CW, Ha } \\
\text { h) Petry I, Egli JB, } \\
\text { i) Israr B, Frazier } \\
\text { j) Petry N, Egli I, } \\
\text { k) Thavarajah P, T } \\
\text { l) Máñez G, Aleg } \\
\text { m) Kan L, Nie S, H } \\
\text { n) Drakou M, Birn } \\
\text { o) Hernández-Sala } \\
\text { p) Magalhaes SC, } \\
\text { q) Ryan E, Galvin } \\
\text { r) Ombra MN, d'A } \\
\text { s) Xu B and Chan } \\
\text { t) Dellavalle DM, }\end{array}$ & $\begin{array}{l}\text { Rengel Z. Food } \\
\text { itriou T, Walcz } \\
\text { hin R, Zimmer } \\
\text { Reddy K. J Exp } \\
\text { dawy T. J Food } \\
\text { edleb K. J Trac } \\
\text { III C, Tulbek M } \\
\text { ahutu PL, Tugir } \\
\text { and Gordon I } \\
\text { ampion B, Niel } \\
\text { varajah D, Van } \\
\text { A, Farre R, Fri } \\
\text {, Wang S, Cui } \\
\text { a A, Koutelidal } \\
\text { ar M, Osorio-I } \\
\text { veira M, Cabri } \\
\text { O'Connor TP, } \\
\text { ierno A, Nazzz } \\
\text { K. Food Chem } \\
\text { andenberg A, }\end{array}$ & $\begin{array}{l}\text { lem 2016; 212: } \\
\text { T, Hurrell RF. B } \\
\text { nn B, Greiner R } \\
\text { t 2015; 66: } 568 \\
\text { mpost Anal } 20 \\
\text { lem Med Biol } 2 \\
\text { lendis M, Heck } \\
\text { ana E, Boy R Hc } \\
\text { l. Food Chem 2 } \\
\text { E E, Hurrell R. J } \\
\text { nberg A. J Agric } \\
\text { a A. Int J Food } \\
\text { V, Li Y, Xu S, W } \\
\text { AE, Komaitis M } \\
\text { z P, Loarca-Piñ } \\
\text { AR, Fonseca AJ, } \\
\text { gguire AR, O'Br } \\
\text { F, Riccardi R, S } \\
\text { 12; } 134: 1287-1 \\
\text { hn RP. J Agric F }\end{array}$ & $\begin{array}{l}\text { 7-433. } \\
\text { J Nutr 2001; 85: 59-63 } \\
\text { nd Roth S. Anim Feed } \\
-5690 . \\
\text { 6; 19: 806-812. } \\
\text { 6; 37: 69-77. } \\
\text { Ogunyemi S. J Sci Fo } \\
\text { ell. J Nutr 2014; 144: } \\
\text { 13; 141: 1690-1693. } \\
\text { Jutr 2013; 143: 1219-1 } \\
\text { ood Chem 2009; 57: } \\
\text { i Nutr 2002; 53: 503-5 } \\
\text { Y, Wang J, Bai Z and X } \\
\text { Panagou EZ, Kapsokef } \\
\text { G, Reynoso-Camacho } \\
\text { /alentao P, Andrade PE } \\
\text { n NM. Plant Foods Hu } \\
\text { igno P, Zaccardelli M, } \\
96 . \\
\text { od Chem 2013; 61: } 80\end{array}$ & $\begin{array}{l}\text { Sci and Technol } 200 \\
\text { od Agric 2015; 95: } 2 \\
681-1687 . \\
24 . \\
044-9049 . \\
08 . \\
\text { ie M. Food Chem To) } \\
\text { lou M. Int J Food Sci } \\
\text { R, Tovar J, Bello-Pére } \\
\text { Food Chem 2017; } 2 \\
\text { m Nutr 2007; 62: } 85 \\
\text { Pane C, Maione M, C } \\
\text { 44-8089. }\end{array}$ & $\begin{array}{l}\text {;33: 320-334. } \\
\text { 87-2291. } \\
\text { col 2017; 467- } \\
\text { Nutr 2016; 66: } \\
\text { LA. J Sci Food } \\
\text { 5: 177-184. } \\
\text { 1. } \\
\text { xid Med Cell Lc }\end{array}$ & $\begin{array}{l}\text { 77-202. } \\
\text { Agric 2010; 90: } \\
\text { gev 2016; } 2016\end{array}$ & 1398298. \\
\hline
\end{tabular}

\section{Índice glicémico (IG)}

El índice glicémico corresponde a un parámetro fisiológico capaz de informar cómo el consumo de un alimento afecta la homeostasis de la glucosa del individuo. Se espera que, tras consumir carbohidratos de la dieta, la glicemia se eleve transitoriamente hasta por dos horas, un fenómeno característico del período postprandial. Un estudio sistemático del IG realizado en $~ 1.100$ individuos enfrentados al consumo de 780 alimentos diferentes, permitió categorizar al consumo de legumbres como un alimento de bajo $I^{36}$. Estos valores han sido estandarizados en referencia al efecto de una masa equivalente de glucosa y han servido como la base para investigar la incorporación de legumbres en la dieta, ya sea como suplemento, harina u otros insumos, sobre parámetros sistémicos durante el período postprandial ${ }^{37,38}$. Un estudio en sujetos sanos, aleatorio y controlado con un grupo placebo, evaluó la ingesta de una preparación alimenticia basada en un extracto de porotos (1,5 - 3 g/día) sobre índices postprandiales como la glicemia, la insulina y la proteína reactiva C. Estos parámetros mostraron valores significativamente menores comparados al grupo control y, además, se reportó un efecto positivo en la saciedad de 
los individuos que ingirieron el extracto ${ }^{39}$. Otros estudios también han explorado la incorporación de harinas de garbanzos en el desayuno, con resultados positivos pero discretos, que apuntan a un mejor control de la glicemia de los sujetos que consumieron pan elaborado con harina de garbanzos ${ }^{40}$. En cualquier caso, la combinación de legumbres con otros alimentos ha sido evaluada con resultados positivos en la respuesta glicémica de los individuos, lo que permite establecer recomendaciones basadas en evidencia obtenida en un escenario alimentario realista ${ }^{41}$.

Algunos estudios han explorado los efectos metabólicos no-inmediatos de la ingesta de legumbres. Nilsson y sus colaboradores ${ }^{42}$, evaluaron el efecto de la ingesta de legumbres pasadas 11-14 horas en dos grupos de individuos sanos, uno que incorporó una ración de porotos $(50 \mathrm{~g})$ al final del día mientras que el otro, consumió una cantidad equivalente en hidratos de carbono en forma de pan blanco. Al día siguiente, tras ingerir un desayuno estándar, el grupo que ingirió la ración de legumbres no sólo experimentó menores alzas de la glucosa e insulina circulantes, sino que también menores niveles de interleuquinas pro-inflamatorias, tales como IL-6 y IL-18, junto con presentar menores niveles de ghrelina y un mayor grado de saciedad ${ }^{42}$. Intervenciones dietarias más largas, sobre 8 semanas, han sido evaluadas en poblaciones caucásicas y asiáticas, entre grupos de individuos sanos y con diagnóstico de diabetes. En todos los reportes, la inclusión de legumbres como parte de un estilo dietario más saludable, apuntan a una mejora cuantificable en marcadores sistémicos como la hemoglobina glicosilada (HbA1c) y el LDL circulante, entre otros ${ }^{43,44}$.

El efecto de las legumbres sobre la respuesta glicémica observada se explica por el tipo de carbohidratos ingeridos. La amilosa, que alcanza un 30 a $40 \%$ de los almidones, está combinada con almidones resistentes a la acción enzimática del intestino delgado y en consecuencia, proceden hacia el intestino grueso donde ocurren los procesos de fermentación. Además, la presencia de ácido fítico interfiere con la actividad de enzimas encargadas de degradar los polímeros de carbohidratos, tales como la $\alpha$-glucosidasa y $\alpha$-amilasa ${ }^{45}$.

\section{Apetito y saciedad}

La saciedad inducida por los alimentos se entiende como el tiempo que le toma al individuo estar dispuesto a ingerir alimentos nuevamente. En un estudio que evaluó el hambre, saciedad y plenitud en 28 individuos adultos y sanos (14 mujeres y 14 hombres) luego de ingerir preparaciones isocalóricas: en base a carne ( $26 \mathrm{~g}$ proteína/3 g fibra) y otra en base a legumbres (17 g proteína/12 g fibra); señala que los individuos que recibieron la comida en base a legumbres reportaron mayor plenitud, saciedad y menos hambre transcurridos 15 minutos del ensayo, la tendencia se mantuvo incluso transcurridas 3 horas $^{46}$. Esta sensación podría estar vinculada a una disminución en la secreción de ghrelina inducida por las legumbres, como lo reporta un estudio en humanos ( 6 mujeres y 6 hombres) sometidos a una comida estándar comparada con una tableta de extracto de poroto ${ }^{47}$. Otras observaciones confirman que una comida basada en legumbres evoca menores niveles de ghrelina comparada con comidas convencionales, y además, aumenta la secreción de péptido YY (PYY) y GLP-2, ambas hormonas consideradas anorexigénicas ${ }^{43}$. Probablemente, la interacción de las legumbres con el colon está estrechamente vinculada a este efecto global, ya que los ácidos grasos de cadena corta producidos por la fermentación microbiana se asocian con un aumento en la expresión de proglucagón, PYY, y la liberación de GLP-1 en un modelo animal ${ }^{47,48}$.

\section{Enfermedades cardiovasculares (ECV)}

Según los indicadores de salud del año 2010, la morbimortalidad por ECV ocupa el primer lugar en Chile, con una tasa de mortalidad observada de 148 por cada 100.000 habitantes $^{49}$. La incorporación de legumbres en la dieta es altamente recomendable para disminuir la prevalencia de ECVs. Un meta-análisis compuesto por 14 estudios que combinó los resultados obtenidos de 367.000 sujetos, permitió observar una disminución del $10 \%$ en la incidencia para enfermedades cardiovasculares en el grupo de sujetos que consumían legumbres (1 porción/día o 3-4 porciones/semana). Al estratificar los tipos de ECV, el consumo de legumbres fue asociado con una disminución del riesgo a padecer cardiopatías coronarias, no así en el riesgo a padecer accidentes cerebrovasculares ${ }^{50}$. Estos resultados concuerdan con el efecto protector del consumo de fibra, cuyo contenido es importante en las legumbres, por cada $7 \mathrm{~g} /$ día de fibra ingerida el riesgo de padecer enfermedades cardio- y cerebro-vasculares disminuye un $9 \%{ }^{51}$.

La relación inversa entre consumo de legumbres y riesgo cardiovascular podría ser explicada en parte por la disminución en los niveles séricos totales de colesterol y LDL encontrados en sujetos que consumen legumbres distintas al poroto soya ${ }^{52}$. Por ejemplo, individuos sometidos a una dieta que incluye $140 \mathrm{~g}$ de garbanzos/día provocó reducciones significativas en el colesterol total sérico y el colesterol asociado a lipoproteínas de baja densidad (LDL-C) comparado con los sujetos sometidos a una dieta basada en trigo y cereales por 5 semanas $^{53}$. Interesantemente, el consumo de alimentos con índice glicémico bajo no es suficiente para provocar la disminución en estos factores de riesgo, la incorporación de fibra en la dieta es un requisito importante para inducir un mejor control de la lipidemia ${ }^{54}$. Estudios con animales de experimentación han arrojado algunas luces sobre los mecanismos que podrían estar operando en el control de la homeostasis de lípidos a nivel sistémico. Grupos de ratas fueron alimentadas con 3 tipos de dietas: una normal, alta en grasas y alta en grasa suplementada con garbanzos. Transcurridos 8 meses de tratamiento, el grupo de animales que recibió el suplemento con garbanzos presentó menor abundancia de tejido adiposo visceral, mejores valores en el perfil lipídico y menor resistencia a insulina comparado con el grupo que 
recibió la dieta alta en grasas solamente. Además, los autores determinaron una mayor actividad de la lipoproteína lipasa en aquellas ratas con dieta alta en grasas y suplementada con garbanzos, lo que es consistente con una disminución en la concentración de triglicéridos en músculo e hígado ${ }^{55}$. En resumen, existe evidencia que apoya la noción de un beneficio cardiovascular por consumo regular de legumbres, un efecto que podría estar mediado por disminución del contenido sérico de colesterol libre y asociado a proteínas.

\section{Microbiota}

La microbiota intestinal está constituida por trillones de bacterias que colectivamente poseen 150 veces más genes que el genoma humano. Cada individuo adquiere su microbiota al nacer y lo acompañará durante el curso de su vida. En términos funcionales, se comporta como un organismo multicelular capaz de interactuar de diversas maneras con el huésped, su composición es dinámica y obedece al estado nutricional y metabólico del huésped ${ }^{56}$.

La mayoría de los elementos de difícil digestión tales como los polímeros de celulosa, correspondientes a las paredes celulares, fibra y carbohidratos resistentes a la digestión, entran a un proceso de fermentación anaeróbica en el intestino grueso, necesario para liberar muchos de los metabolitos atrapados en la estructura vegetal y producir ácidos grasos de cadena corta (acetato, propionato, butirato, entre otros $)^{57}$. Estos últimos son esenciales para la bioenergética de las células epiteliales del intestino ${ }^{58} y$ pueden establecer un $\mathrm{pH}$ en el lumen del intestino grueso proximal cercano a 5,5-6,5, condiciones que protegen de la colonización de bacterias patogénicas como Salmonella spp. y Escherichia Coli59.

En efecto, la fermentación de semillas pre-digeridas de habas (Vicia faba) y lupino (Lupinus albus) en cultivos anaeróbicos generó niveles importantes de ácidos grasos de cadena corta cercanos a $81 \mathrm{mM}$ (lupino) y $78 \mathrm{mM}$ (habas). Los grupos microbianos cuya abundancia fue favorecida significativamente fueron Bifidobacterium spp., Lactobacillus-Enterococcus, Atopobium, BacteroidesPretovella, Clostridium coccoides-Eubacterium rectale, Faecalibacterium prausnitzii and Roseburia intestinalis ${ }^{60}$. Este impacto sobre la flora bacteriana sugiere que las legumbres constituyen ingredientes atractivos para el diseño de nuevos alimentos funcionales, dirigidos a promover la salud humana a través de la prevención del desarrollo de desórdenes metabólicos y gastrointestinales que incluyen síndrome de intestino irritable, obesidad y diabetes, entre otras ${ }^{61}$.

\section{Cáncer colo-rectal}

Los cánceres digestivos en su conjunto son responsables del $41,8 \%$ del total de los fallecimientos por cáncer. El cáncer colo-rectal es la segunda causa de muerte después del cáncer gástrico ${ }^{49}$, con un 5,9\% del total de las muertes por cáncer.

Existe evidencia de una relación positiva entre el consumo de legumbres y la disminución del riesgo asociado al cáncer colo-rectal. Una cohorte de 2.818 individuos seguida por 26 años concluyó que el consumo de legumbres con una frecuencia de 3 veces/semana disminuye el riesgo de presentar pólipos colo-rectales $\left(\mathrm{OR}\right.$ 0,67) ${ }^{62,63}$. Complementario a esta observación, un meta-análisis comprendido por 32 artículos que analizó la relación entre patrones dietéticos y el riesgo de padecer cáncer colo-rectal y adenomas, indicó que patrones dietéticos saludables que incluyen el consumo regular de frutas, verduras, alimentos bajos en grasa, pescados, aves y legumbres se asocia con una disminución en el riesgo de padecer cáncer colo-rectal de (OR 0,45 a 0,90). Un resultado totalmente opuesto fue encontrado al observar la asociación de patrones dietéticos tradicionales que incluyen consumo de carnes procesadas, papas, alimentos altos en grasas y bajo consumo de frutas y legumbres, el cual se asoció con un aumento del riesgo de cáncer colo-rectal (OR 1,18 a 11,7) ${ }^{64}$.

Modelos animales y celulares sugieren que las saponinas contenidas en las legumbres podrían estar mediando este efecto protector sobre el epitelio intestinal. En particular, una dieta suplementada con harina de garbanzo $(10 \%)$ por 10 semanas fue efectiva para disminuir un $64 \%$ la formación de focos aberrantes de criptas colónicas en un modelo murino de tumores inducidos ${ }^{65,66}$. Experimentalmente, la incubación con apigenina $(0-50 \mu \mathrm{M})$ por 24 horas en células HCT116 resultó en el arresto celular de esta línea celular, un efecto mediado por el aumento en la expresión de proteínas p53, p21CIP1 y WAF1, las cuales inhiben a la quinasa dependiente de ciclina ${ }^{30,67}$. La inhibición de la proliferación también ha sido observada en células Caco-2 tratadas por 48 horas a distintos extractos hidrofílicos de legumbres, siendo el extracto de lentejas el que exhibió el mayor efecto antiproliferativo ${ }^{68}$. Otros estudios han reportado que la apigenina $(6,25$ a $50 \mu \mathrm{M})$ provoca condensación cromosómica y formación de cuerpos apoptóticos, rasgos característicos de muerte celular programada, lo cual fue confirmado por la activación de caspasas y corte proteolítico de PARP ${ }^{30}$.

\section{CONCLUSIONES}

La compilación de datos presentados en este trabajo permite actualizar la información respecto de los efectos beneficiosos que podrían resultar del consumo habitual de legumbres. Transitar desde la descripción del contenido en macro y micro-nutrientes de especies de legumbres hacia los mecanismos fisiológicos involucrados por la metabolización de sus constituyentes, permite identificar a la fermentación colónica, como un proceso crucial para mantener una relación saludable entre la flora intestinal y la barrera epitelial. La generación de ácidos grasos de cadena corta, con la concomitante acidificación del lumen intestinal, son condiciones claves que evitan la colonización por bacterias de carácter patogénico y no menos importante, es que la diversidad microbiana en conjunto con los metabolitos generados in situ promueven un balance de la respuesta inmunológica debido a la 
cercanía con células inmune residentes del epitelio intestinal (Figura 1).

Durante el tránsito intestinal se produce la liberación de fitoesteroles y flavonoides, como la apigenina, que podrían controlar la proliferación descontrolada de colonocitos y así, evitar la aparición de pólipos y eventualmente, disminuir la prevalencia del cáncer colo-rectal. En términos metabólicos, es indudable que la degradación pausada y tardía de almidones resistentes a la digestión, también induce una respuesta integral del sistema gastrointestinal para coordinar no sólo la sensación de saciedad y placer, que debe acompañar a la ingesta de una comida satisfactoria, sino que además promueve la liberación sistémica de hormonas que modulan el uso y, en consecuencia, el tiempo de permanencia de la glucosa circulante (Figura 1).
Es interesante destacar que procesos básicos comúnmente empleados durante la preparación de las legumbres no afectan mayormente su composición y naturalmente sus beneficios se mantienen. Esta observación ha alentado el estudio de preparaciones que incorporan harinas o suplementos derivados de legumbres en presentaciones alimentarias cotidianas, como el pan. Este tipo de iniciativas deberían ser exploradas con mayor interés por la industria, particularmente porque las legumbres ofrecen altos contenidos de proteínas y fibra que hoy en día enfrentan una demanda creciente por parte de una población cada vez más exigente. De hecho, la inclusión de harinas derivadas de legumbres ha sido evaluada en diferentes entornos, sin embargo, mayores esfuerzos deberían estar orientados a segmentos de la población, que por sus estilos de vida no

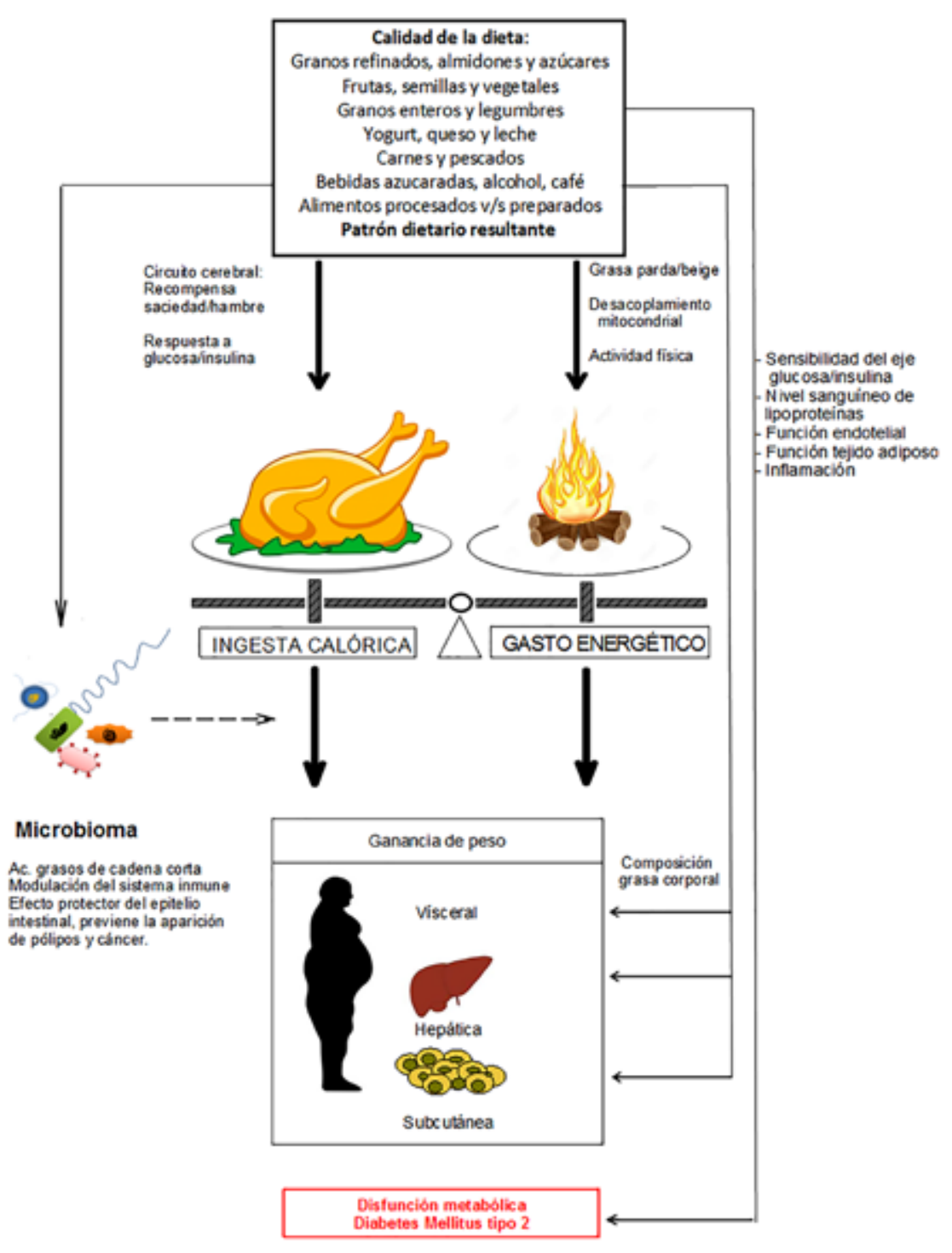


tan saludables, caen en riesgo de padecer patologías cerebro y cardio-vasculares. En términos económicos, las pérdidas por enfermedades crónicas se estiman cercanas a los $\$ 17$ trillones de dólares entre el 2011 y el 2030, por lo que promover su consumo de manera activa en la población corresponde a una iniciativa perfectamente alineada con políticas públicas que mejoren el bienestar de la población ${ }^{69}$.

Es evidente que el consumo de legumbres no sólo es recomendable desde la perspectiva de los beneficios fisiológicos a los que podría optar un individuo al consumirlos, sino también porque al incluirlos en la dieta de forma regular comienza a operar un círculo virtuoso que conduce a una disminución en la ingesta de productos ultra-procesados. En términos culturales, el ejercicio de preparar y consumir legumbres reaviva la presencia de platos tradicionales, de manera tal que la matriz productiva se orienta hacia cultivos cuya producción está en armonía con un principio de sustentabilidad medioambiental. A modo de ejemplo, la implementación de una dieta mediterránea, que promueve entre otras cosas, la sustitución de la carne por legumbres o pescado, constituye una disminución importante en el impacto ecológico, la huella de carbono y agua asociados con su producción ${ }^{70}$.

\section{BIBLIOGRAFÍA}

1. Zohary D, Hopf M. Domestication of Pulses in the Old World: Legumes were companions of wheat and barley when agriculture began in the Near East. Science 1973; 182: 887894.

2. Imamura F, Micha R, Khatibzadeh S, Fahimi S, Shi P, Powles J et al. Dietary quality among men and women in 187 countries in 1990 and 2010: a systematic assessment. Lancet Glob Health 2015; 3: e132-e142.

3. Office of Agricultural Studies and Policies M.d.A.G.d.C. Historical annual crops 2016; 22-8-2016.

4. Office of Agricultural Studies and Policies M.d.A.G.d.C. Historical annual crops. Apparent consumption of main food in Chile. 2012. 2016.

5. Kan L, Nie S, Hu J, Wang S, Cui SW, Li Y et al. Nutrients, phytochemicals and antioxidant activities of 26 kidney bean cultivars. Food Chem Toxicol 2017; 467-477.

6. Du SK, Jiang H, Ai Y, Jane JL. Physicochemical properties and digestibility of common bean (Phaseolus vulgaris L.) starches. Carbohydr Polym. 2014; 108:200-205.

7. Duranti M. Grain legume proteins and nutraceutical properties. Fitoterapia 2006; 77: 67-82.

8. Clemente $A$, Carmen Marin-Manzano M, Jimenez E, Carmen $A M$, Domoney C. The anti-proliferative effect of TI1B, a major Bowman-Birk isoinhibitor from pea (Pisum sativum L.), on HT29 colon cancer cells is mediated through protease inhibition. Br J Nutr 2012; 108 Suppl 1: S135-S144.

9. Luna-Vital DA, Gonzalez de ME, Mendoza S, Loarca-Pina G. Peptides present in the non-digestible fraction of common beans (Phaseolus vulgaris L.) inhibit the angiotensin-l converting enzyme by interacting with its catalytic cavity independent of their antioxidant capacity. Food Funct 2015; 6: 1470-1479.

10. Hernandez-Salazar M, Osorio-Diaz P, Loarca-Pina G, ReynosoCamacho R, Tovar J, Bello-Perez LA. In vitro fermentability and antioxidant capacity of the indigestible fraction of cooked black beans (Phaseolus vulgaris L.), lentils (Lens culinaris L.) and chickpeas (Cicer arietinum L.). I Sci Food Agric 2010; 90: 1417-1422.

11. Surampudi P, Enkhmaa B, Anuurad E, Berglund L. Lipid Lowering with Soluble Dietary Fiber. Curr Atheroscler Rep 2016; 18: 75 .

12. Mudgil D, Barak S. Composition, properties and health benefits of indigestible carbohydrate polymers as dietary fiber: a review. Int J Biol Macromol 2013; 61: 1-6.

13. McRorie JW, Chey WD. Fermented Fiber Supplements Are No Better Than Placebo for a Laxative Effect. Dig Dis Sci 2016; 61: 3140-3146.

14. McRorie JW Jr, McKeown NM. Understanding the Physics of Functional Fibers in the Gastrointestinal Tract: An EvidenceBased Approach to Resolving Enduring Misconceptions about Insoluble and Soluble Fiber. I Acad Nutr Diet 2017; 117: 251-264.

15. Tian L, Bruggeman G, van den Berg M, Borewicz K, Scheurink AJ, Bruininx E et al. Effects of pectin on fermentation characteristics, carbohydrate utilization, and microbial community composition in the gastrointestinal tract of weaning pigs. Mol Nutr Food Res 2017; 61(1).

16. Satoh H, Urushidani T. Soluble Dietary Fiber Can Protect the Gastrointestinal Mucosa Against Nonsteroidal Anti-Inflammatory Drugs in Mice. Dig Dis Sci 2016; 61: 1903-1914.

17. Hino S, Sonoyama K, Bito H, Kawagishi H, Aoe S, Morita T. Low-methoxyl pectin stimulates small intestinal mucin secretion irrespective of goblet cell proliferation and is characterized by jejunum Muc2 upregulation in rats. J Nutr 2013; 143: 34-40.

18. Sandberg AS, Brune M, Carlsson NG, Hallberg L, Skoglund $E$, Rossander-Hulthen L. Inositol phosphates with different numbers of phosphate groups influence iron absorption in humans. Am J Clin Nutr 1999; 70: 240-246.

19. Petry N, Egli I, Gahutu JB, Tugirimana PL, Boy E, Hurrell R. Phytic acid concentration influences iron bioavailability from biofortified beans in Rwandese women with low iron status. J Nutr 2014; 144: 1681-1687.

20. Dellavalle DM, Vandenberg A, Glahn RP. Seed coat removal improves iron bioavailability in cooked lentils: studies using an in vitro digestion/Caco-2 cell culture model. J Agric Food Chem 2013; 61: 8084-8089.

21. Thavarajah $D$, Thavarajah $P$, Sarker A, Vandenberg A. Lentils (Lens culinaris Medikus Subspecies culinaris): a whole food for increased iron and zinc intake. J Agric Food Chem 2009; 57: 5413-5419.

22. Saleh A Alajaji, Tarek A El-Adawy. Nutritional composition of chickpea (Cicer arietinum L.) as affected by microwave cooking and other traditional cooking methods. Journal of Food Composition and Analysis 2006; 19: 806-812.

23. Drakou M, Birmpa A, Koutelidakis AE, Komaitis M, Panagou EZ, Kapsokefalou M. Total antioxidant capacity, total phenolic content and iron and zinc dialyzability in selected Greek varieties of table olives, tomatoes and legumes from conventional and organic farming. Int J Food Sci Nutr 2015; 66: 197-202.

24. Ombra MN, d'Acierno A, Nazzaro F, Riccardi R, Spigno $P$, Zaccardelli $M$ et al. Phenolic Composition and Antioxidant and Antiproliferative Activities of the Extracts of Twelve Common Bean (Phaseolus vulgaris L.) Endemic Ecotypes of Southern Italy before and after Cooking. Oxid Med Cell Longev 2016; 2016: 1398298.

25. Magalhaes SC, Taveira M, Cabrita AR, Fonseca AJ, Valentao $P$, Andrade PB. European marketable grain legume seeds: Further insight into phenolic compounds profiles. Food Chem 
2017; 215: 177-184.

26. Franke AA, Custer LJ, Hundah/ SA. Determinants for urinary and plasma isoflavones in humans after soy intake. Nutr Cancer 2004; 50: 141-154.

27. Prince $P D$, Lanzi $C R$, Toblli JE, Elesgaray $R$, Oteiza PI, Fraga $C G$ et al. Dietary (-)-epicatechin mitigates oxidative stress, NO metabolism alterations, and inflammation in renal cortex from fructose-fed rats. Free Radic Biol Med 2016; 90: 35-46.

28. Farhan M, Zafar A, Chibber S, Khan HY, Arif H, Hadi SM. Mobilization of copper ions in human peripheral lymphocytes by catechins leading to oxidative DNA breakage: A structure activity study. Arch Biochem Biophys 2015; 580: 31-40.

29. Guttenplan JB, Chen KM, Sun YW, Kosinska W, Zhou Y, Kim $S A$ et al. Effects of Black Raspberry Extract and Protocatechuic Acid on Carcinogen-DNA Adducts and Mutagenesis, and Oxidative Stress in Rat and Human Oral Cells. Cancer Prev Res (Phila) 2016; 9: 704-712.

30. Lee Y, Sung B, Kang YJ, Kim DH, Jang JY, Hwang SY et al. Apigenin-induced apoptosis is enhanced by inhibition of autophagy formation in HCT116 human colon cancer cells. Int J Oncol 2014; 44: 1599-1606.

31. Cao X, Liu B, Cao W, Zhang W, Zhang $F$, Zhao $H$ et al. Autophagy inhibition enhances apigenin-induced apoptosis in human breast cancer cells. Chin J Cancer Res 2013; 25: 212-222.

32. Ryan E, Galvin K, O'Connor TP, Maguire AR, O'Brien NM. Phytosterol, squalene, tocopherol content and fatty acid profile of selected seeds, grains, and legumes. Plant Foods Hum Nutr 2007; 62: 85-91.

33. Plat J, Baumgartner S, Mensink RP. Mechanisms Underlying the Health Benefits of Plant Sterol and Stanol Ester Consumption. J AOAC Int 2015; 98: 697-700.

34. Neil HA, Meijer GW, Roe LS. Randomised controlled trial of use by hypercholesterolaemic patients of a vegetable oil sterol-enriched fat spread. Atherosclerosis 2001; 156: 329-337.

35. Shepherd J, Cobbe SM, Ford I, Isles CG, Lorimer AR, MacFarlane PW et al. Prevention of coronary heart disease with pravastatin in men with hypercholesterolemia. West of Scotland Coronary Prevention Study Group. N Engl I Med. 1995; 333: 1301-1307.

36. Brand-Miller JC, Stockmann K, Atkinson F, Petocz P, Denyer G. Glycemic index, postprandial glycemia, and the shape of the curve in healthy subjects: analysis of a database of more than 1.000 foods. Am J Clin Nutr 2009; 89: 97-105.

37. Anderson GH, Liu Y, Smith CE, Liu TT, Nunez MF, Mollard $R C$ et al. The acute effect of commercially available pulse powders on postprandial glycaemic response in healthy young men. Br J Nutr. 2014; 112:1966-973.

38. Ramdath D, Renwick S, Duncan AM. The Role of Pulses in the Dietary Management of Diabetes. Can J Diabetes 2016; 40: 355-363.

39. Spadafranca A, Rinelli S, Riva A, Morazzoni P, Magni P, Bertoli $S$ et al. Phaseolus vulgaris extract affects glycometabolic and appetite control in healthy human subjects. Br I Nutr 2013; 109: 1789-1795.

40. Johnson SK, Thomas SJ, Hall RS. Palatability and glucose, insulin and satiety responses of chickpea flour and extruded chickpea flour bread eaten as part of a breakfast. Eur J Clin Nutr 2005; 59: 169-76.

41. Thompson SV, Winham DM, Hutchins AM. Bean and rice meals reduce postprandial glycemic response in adults with type 2 diabetes: a cross-over study. Nutr J 2012; 11: 23.

42. Nilsson A, Johansson E, Ekstrom L, Bjorck I. Effects of a brown beans evening meal on metabolic risk markers and appetite regulating hormones at a subsequent standardized breakfast: a randomized cross-over study. PLoS One 2013; 8: e59985.

43. Jenkins DJ, Kendall CW, Augustin LS, Mitchell S, Sahye-Pudaruth $S$, Blanco MS et al. Effect of legumes as part of a low glycemic index diet on glycemic control and cardiovascular risk factors in type 2 diabetes mellitus: a randomized controlled trial. Arch Intern Med 2012; 172: 1653-1660.

44. Zhou M, Zhu L, Cui X, Feng L, Zhao X, He S et al. Influence of diet on leukocyte telomere length, markers of inflammation and oxidative stress in individuals with varied glucose tolerance: a Chinese population study. Nutr J 2016; 15: 39.

45. Sparvoli F, Laureati M, Pilu R, Pagliarini E, Toschi I, Giuberti $G$ et al. Exploitation of Common Bean Flours with Low Antinutrient Content for Making Nutritionally Enhanced Biscuits. Front Plant Sci 2016; 7: 928.

46. Bonnema AL, Altschwager D, Thomas W, Slavin JL. The Effects of a Beef-Based Meal Compared to a Calorie Matched BeanBased Meal on Appetite and Food Intake. J Food Sci 2015; 80: H2O88-H2O93.

47. Tolhurst $G$, Heffron H, Lam YS, Parker HE, Habib AM, Diakogiannaki $E$ et al. Short-chain fatty acids stimulate glucagon-like peptide-1 secretion via the G-protein-coupled receptor FFAR2. Diabetes 2012; 61: 364-371.

48. Zhou J, Keenan MJ, Fernandez-Kim SO, Pistell PJ, Ingram DK, $L i B$ et al. Dietary resistant starch improves selected brain and behavioral functions in adult and aged rodents. Mol Nutr Food Res 2013; 57: 2071-2074.

49. Ministerio de Salud-Gobierno de Chile. National Health Survey. 2009-2010.

50. Marventano S, Izquierdo PM, Sanchez-Gonzalez C, Godos J, Speciani A, Galvano $F$ et al. Legume consumption and CVD risk: a systematic review and meta-analysis. Public Health Nutr 2017; 20: 245-254.

51. Threapleton DE, Greenwood DC, Evans CE, Cleghorn CL, Nykjaer C, Woodhead C et al. Dietary fibre intake and risk of cardiovascular disease: systematic review and meta-analysis. BMJ 2013; 347: f6879.

52. Bazzano LA, Thompson AM, Tees MT, Nguyen CH, Winham DM. Non-soy legume consumption lowers cholesterol levels: a meta-analysis of randomized controlled trials. Nutr Metab Cardiovasc Dis 2011; 21: 94-103.

53. Pittaway JK, Ahuja KD, Cehun M, Chronopoulos A, Robertson IK, Nestel PJ et al Dietary supplementation with chickpeas for at least 5 weeks results in small but significant reductions in serum total and low-density lipoprotein cholesterols in adult women and men. Ann Nutr Metab. 2006; 50:512-518.

54. Goff LM, Cowland DE, Hooper L, Frost GS. Low glycaemic index diets and blood lipids: a systematic review and metaanalysis of randomised controlled trials. Nutr Metab CardiovasC Dis 2013; 23: 1-10.

55. Yang Y, Zhou L, Gu Y, Zhang Y, Tang J, Li F et al. Dietary chickpeas reverse visceral adiposity, dyslipidaemia and insulin resistance in rats induced by a chronic high-fat diet. Br J Nutr 2007; 98: 720-726.

56. Ramakrishna BS. Role of the gut microbiota in human nutrition and metabolism. J Gastroenterol Hepatol 2013; 28 Suppl 4:9-17.

57. Flint HJ, Bayer EA, Rincon MT, Lamed R, White BA. Polysaccharide utilization by gut bacteria: potential for new insights from genomic analysis. Nat Rev Microbiol 2008; 6: 121-131.

58. Hamer HM, Jonkers D, Venema K, Vanhoutvin S, Troost FJ, 
Brummer RJ. Review article: the role of butyrate on colonic function. Aliment Pharmacol Ther 2008, 27: 104-119.

59. Duncan $\mathrm{SH}$, Louis $P$, Thomson JM, Flint HJ. The role of $\mathrm{pH}$ in determining the species composition of the human colonic microbiota. Environ Microbiol 2009; 11: 2112-2122.

60. Gullon P, Gullon B, Tavaria F, Vasconcelos M, Gomes AM. In vitro fermentation of lupin seeds (Lupinus albus) and broad beans (Vicia faba): dynamic modulation of the intestinal microbiota and metabolomic output. Food Funct 2015; 6: 3316-3322.

61. Belkaid Y, Hand TW. Role of the microbiota in immunity and inflammation. Cell 2014; 157: 121-141.

62. Tantamango YM, Knutsen SF, Beeson L, Fraser G, Sabate J. Association between dietary fiber and incident cases of colon polyps: the adventist health study. Gastrointest Cancer Res 2011; 4: 161-167.

63. Tantamango YM, Knutsen SF, Beeson WL, Fraser G, Sabate J. Foods and food groups associated with the incidence of colorectal polyps: the Adventist Health Study. Nutr Cancer 2011; 63: 565-572.

64. Randi G, Edefonti V, Ferraroni M, La VC, Decarli A. Dietary patterns and the risk of colorectal cancer and adenomas.
Nutr Rev 2010; 68: 389-408.

65. Murillo G, Choi JK, Pan O, Constantinou Al, Mehta RG. Efficacy of garbanzo and soybean flour in suppression of aberrant crypt foci in the colons of CF-1 mice. Anticancer Res 2004; 24: 3049-3055.

66. Guo YW, Chen YH, Chiu WC, Liao H, Lin SH. Soy Saponins Meditate the Progression of Colon Cancer in Rats by Inhibiting the Activity of beta-Glucuronidase and the Number of Aberrant Crypt Foci but Not Cyclooxygenase-2 Activity. ISRN Oncol 2013; 2013: 645817.

67. Sung B, Chung HY, Kim ND. Role of Apigenin in Cancer Prevention via the Induction of Apoptosis and Autophagy. I Cancer Prev 2016; 21: 216-226.

68. Xu B, Chang SK. Comparative study on antiproliferation properties and cellular antioxidant activities of commonly consumed food legumes against nine human cancer cell lines. Food Chem 2012; 134: 1287-1296.

69. Mozaffarian D. Dietary and Policy Priorities for Cardiovascular Disease, Diabetes, and Obesity: A Comprehensive Review. Circulation 2016; 133: 187-225.

70. Fara GM. Nutrition between sustainability and quality. Ann Ig 2015; 27: 693-704. 